\title{
Long-term Outcome of Infants with Spina Bifida Through Assessment of the Prognostic Value of Hostile Bladder Parameters
}

\author{
(1) Ali Tekin, (1) Sibel Tiryaki, (1) Ezgi Altun Tanıl, @ibrahim Ulman \\ Ege University Faculty of Medicine Hospital, Clinic of Pediatric Surgery, Division of Pediatric Urology, İzmir, Turkey
}

\begin{abstract}
Aim: In 2016, the Centers for Disease Control and Prevention published a management algorithm for Spina Bifida (SB) cases from birth and started collecting data prospectively. They designated risk factors from urodynamic studies as end filling pressure or detrusor leak point pressure (DLPP) $\geq 40 \mathrm{cmH}_{2} \mathrm{O}$ or neurogenic detrusor overactivity (NDO) with detrusor sphincter dyssynergia (DSD), and categorized this type of bladder dysfunction as "hostile bladder" (HB). They recommended the immediate start of clean intermittent catheterization and anticholinergics in these patients. Having similar concerns regarding this patient population, we designed a retrospective study to identify and reveal the longterm outcomes of SB patients with HB.
\end{abstract}

Materials and Methods: All urodynamic studies and hospital records of SB patients admitted and followed between 1994-2014 were reviewed retrospectively. The demographic data, the presence of DLPP, DSD and NDO in the first urodynamic examination, bladder compliance, first and last radiologic and scintigraphic imagings, and surgical interventions were evaluated. Upper tract damage was defined as new scars in dimercaptosuccinic acid scans.

Results: A total of 58 patients were included in this study. The mean follow-up was $12.17 \pm 5.17$ years. The presence of a scar in the first scintigraphy $(p=0.01)$ and the presence of hydronephrosis in the first and last ultrasonography $(p=0.03)$ were found to be independent risk factors for new scar development. When DLPP values were evaluated with receiver operating characteristic curve analysis, $50 \mathrm{cmH}_{2} \mathrm{O}$ was observed as a significant threshold value with $73 \%$ sensitivity and $60 \%$ specificity.

Conclusion: Our study confirmed the detrimental effects of high pressure and detrusor-sphincter dyssynergia; however, HB parameters were not sufficient to distinguish high-risk group patients. The presence of scars in the first scintigraphic evaluation, DLPP above $50 \mathrm{cmH}_{2} \mathrm{O}$, and the presence of hydronephrosis in the first ultrasound were found to be risk factors for renal deterioration. More frequent monitoring and detailed evaluation may be necessary for patients with these risk factors.

Keywords: Spina Bifida, hostile bladder, neuropathic bladder, detrusor leak point pressure, neurogenic detrusor overactivity

\section{Introduction}

Spina Bifida (SB) is a condition that may result in chronic kidney disease secondary to bladder dysfunction. Despite a worldwide varying incidence, 1-6 cases are seen in every 1,000 live births (1). Several studies reported better results with pro-active management strategies (2,3). Different urodynamic parameters or scoring systems were suggested to detect patients who are at risk of upper urinary tract damage (4-6). In 2016, the Centers for Disease Control and Prevention (CDC) published a management algorithm for SB 
cases from birth and started collecting data prospectively (7). They designated risk factors from urodynamic studies as end filling pressure or detrusor leak point pressure (DLPP) $40 \mathrm{cmH}_{2} \mathrm{O}$ or greater, or neurogenic detrusor overactivity (NDO) with detrusor sphincter dyssynergia (DSD), and categorized this type of bladder dysfunction as "hostile bladder". They recommended the immediate start of clean intermittent catheterization and anticholinergics in these patients. Having similar concerns regarding this patient population in our region, we designed a retrospective study with an aim to identify and reveal the long-term outcomes of SB patients with "hostile bladders".

\section{Materials and Methods}

All urodynamic studies and hospital records of SB patients admitted and followed between 1994 and 2014 were reviewed retrospectively to identify patients with "hostile" bladders according to the above-mentioned criteria. Patients with the first admission after infancy, those who did not comply with the SB follow-up protocol of our department, and those patients followed for less than five years were excluded from this study.Urodynamic tests (pressure-flow study) were performed according to our protocols in all cases using a double-lumen 6 Fr urodynamic catheter by Dyno (AYMED ${ }^{\circledR}$ Istanbul, Turkey). Pelvic floor activity was evaluated with a total of 3 electromyography electrodes, 2 of them placed on the perianal skin (right and left of the anus), and one for reference on the thigh. Intraabdominal pressure was measured using an $8 \mathrm{Fr}$ rectal balloon catheter. At least two fillings were done for each case. The lowest leakage pressure was described as DLPP. DSD was defined as involuntary pelvic floor activation during voiding. The involuntary phasic or terminal detrusor contractions during filling were described as NDO. The demographic data of the cases, the presence of DLPP, DSD and NDO in the first urodynamic examination, bladder compliance, first and last dimercaptosuccinic acid (DMSA) scans, first and last voiding cystourethrograms, surgical interventions, first and last kidney ultrasound, and the medications they received were evaluated. All patients were followed according to the standardized program of our department (Table I). Urodynamic and clinical data, along with the above-defined hostile bladder parameters, were evaluated for predicting upper tract damage. Upper tract damage was defined as new scars in DMSA scans.

\section{Statistical Analysis}

Statistical analysis was done with the IBM SPSS 21 program. Kolmogorov-Smirnov test was used to evaluate the distribution, Pearson chi-square, Mann-Whitney $U$, Kruskal-Wallis, regression analysis, and receiver operator characteristics curve (ROC) tests were used for analysis where appropriate. Institutional Ethical approval was obtained for the retrospective patient chart review (208T/26).

\section{Results}

The flow-chart describes patient selection for the study (Figure 1). A total of 58 patients (33 boys and 25 girls) with "hostile" bladder were included in this study. The mean follow-up was $12.17 \pm 5.17$ years. Hydronephrosis was present in the first ultrasonography (US) in eight cases. It resolved in five cases and was present in a total of 5 cases with two being new-onset cases. Renal scars were observed initially in the first DMSA scans of 10 cases. Nine patients had developed new scars at the last DMSA, making a total of 19 cases with renal parenchymal scars. The mean DLPP value at the first urodynamic study was $54.88 \pm 30.43 \mathrm{cmH}_{2} \mathrm{O}$. Of the 58 patients, DSD was present in $48(82.7 \%)$ in the first and $49(84.5 \%)$ in the last urodynamic study. NDO was present
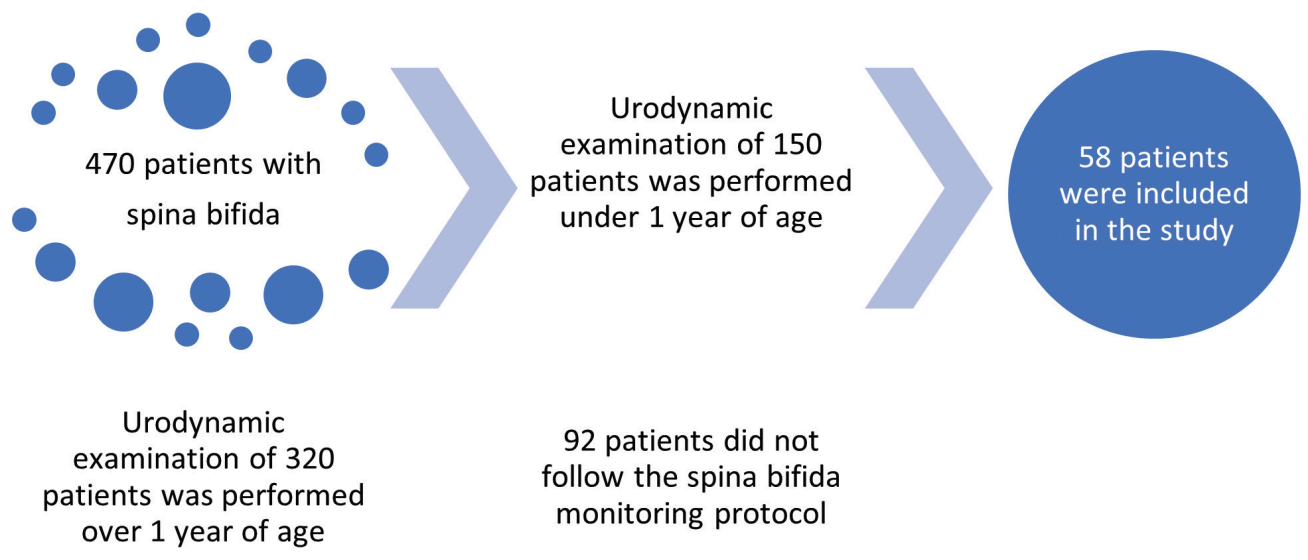

\begin{abstract}
92 patients did not follow the spina bifida monitoring protocol
\end{abstract}

Figure 1. Patient admission flow chart 
in 38 cases (65.5\%) in the first and last urodynamics (with nine of these being new-onset cases in the last and nine others having resolved after the first).

The presence of a scar in the first scintigraphy $(p=0.01)$ and the presence of hydronephrosis in the first and last US $(p=0.03)$ were found to be independent risk factors for new scar development (Table II). When $40 \mathrm{cmH}_{2} \mathrm{O}$ was accepted as the threshold value, no significant relation was found between DLPP and new scar development $(p=0.05)$. When DLPP values were evaluated with ROC analysis for new scar development, $50 \mathrm{cmH}_{2} \mathrm{O}$ was observed as a significant threshold value with $73 \%$ sensitivity and $60 \%$ specificity. A significant correlation was found between this threshold of DLPP and the development of a new scar $(p=0.03)$. Kidney failure was observed in only one case. Bladder augmentation was performed in $7(12.1 \%)$ of the cases. All patients who underwent augmentation cystoplasty had NDO $(p=0.041)$.

Table I. Urological follow-up protocol in Spina Bifida patients

\begin{tabular}{|c|c|c|c|c|c|}
\hline Age & UA \& UC & Urinary US & UD & Scintigraphy & VCUG \\
\hline Preoperative & $x$ & $x$ & & & \\
\hline Postoperative 1 week & $x$ & $x$ & & & \\
\hline 6 week & $x$ & $x$ & $x$ & & \\
\hline 6 months & $x$ & $x$ & $x$ & $x$ & $x$ \\
\hline 1 year & $x$ & $x$ & $x$ & $(X)$ & \\
\hline 1.5 year & $x$ & & & & $(X)$ \\
\hline 2 year & $x$ & $x$ & $x$ & $x$ & \\
\hline 3 year & $x$ & $(X)$ & $(X)$ & & $(X)$ \\
\hline 4 year & $x$ & $x$ & $x$ & & \\
\hline 5 year & $x$ & $(X)$ & $(X)$ & & $(X)$ \\
\hline 6 year & $x$ & $x$ & $x$ & & \\
\hline 7 year & $x$ & $(X)$ & $(X)$ & & $(X)$ \\
\hline 8 year & $x$ & $x$ & $x$ & & \\
\hline 9 year & $x$ & $(X)$ & $(X)$ & & \\
\hline 10 year & $x$ & $x$ & $x$ & & $(X)$ \\
\hline
\end{tabular}

$\mathrm{X}$ : These are mandatory examinations.

$(\mathrm{X})$ : In patients without infection, reflux and urodynamic examination within normal limits, these examinations are optional depending on the clinical situation.

$\mathrm{CIC}$ : CIC training is given to families of all newborn patients. If there is no hydronephrosis and if there is no residual urine in the urodynamic examination at Week 6 ,

$\mathrm{CIC}$ is discontinued.

Anticholinergic therapy is started in patients with the following features:

-NDO

$-D S D$

$-\mathrm{DLPP}>40 \mathrm{cmH}_{2} \mathrm{O}$

-Low compliance

Prophylactic antibiotic is started (amoxicillin or trimethoprim-sulfamethoxazole, depending on the age) in patients with the following features:

-3-6 months after initiation of CIC

-VUR

-Recurrent UTI

UA and UC: Urinalysis and urine culture, US: Ultrasound, UD: Urodynamics, VCUG: Voiding cystourethrogram, CIC: Clean intermittent catheterization, NDO: Neurogenic detrusor overactivity, DSD: Detrusor-sphincter dyssynergia, DLPP: Detrusor leak point pressure, VUR: Vesicoureteral reflux, UTI: Urinary tract infection

Table II. Patient characteristics according to the presence of new-onset scar in the last DMSA

\begin{tabular}{|c|c|c|c|c|c|c|}
\hline $\begin{array}{l}\text { New-onset scar } \\
\text { in the last DMSA }\end{array}$ & $\begin{array}{l}\text { First urodynamic } \\
\text { DLPP value (mean) }\end{array}$ & $\begin{array}{l}\text { HN presence in } \\
\text { the first RBUS }\end{array}$ & $\begin{array}{l}\text { Scar presence in } \\
\text { the first DMSA }\end{array}$ & $\begin{array}{l}\text { DSD Presence } \\
\text { in the first UD }\end{array}$ & $\begin{array}{l}\text { NDO presence } \\
\text { in the first UD }\end{array}$ & $\begin{array}{l}\text { DLPP }>50 \mathrm{cmH}_{2} \mathrm{O} \\
\text { in the first UD }\end{array}$ \\
\hline Positive & $65.40 \pm 40.03^{*}$ & $6^{* *}$ & $9^{* *}$ & $15^{*}$ & $11^{*}$ & $11^{* *}$ \\
\hline Negative & $51.21 \pm 25.88$ & 2 & 1 & 33 & 27 & 17 \\
\hline
\end{tabular}


There was no relation to other variables, such as the presence of vesicoureteral reflux or DSD. The mean DLPP values $\left(63.86 \pm 24.98 \mathrm{cmH}_{2} \mathrm{O}\right)$ in the first urodynamic studies of the patients who required bladder augmentation were higher than in other cases $\left(53.65 \pm 31.12 \mathrm{cmH}_{2} \mathrm{O}\right)$, but it was not significant $(p=0.41)$.

\section{Discussion}

The necessity of urological follow-up is not questioned for patients with SB, but the frequency and design of followup are not well-defined or standardized. Close monitoring and pro-active patient management are shown to reduce upper urinary tract damage and bladder surgery $(2,3)$. The follow-up protocol of our department involves outpatient visits every 6 months until the age of 2, followed by annual visits even in uneventful cases. Long-term followup of all cases is recommended due to the risk of tethered cord and its urological consequences (8). However, our study revealed that only a small number of patients were compliant with this protocol, and only a small group of the patients we followed could be included in the study (flowchart/Figure 1). Many factors, such as mobility restrictions of the patients, or socio-economic problems, may hinder attending the outpatient visits regularly. Identifying patients at risk and arranging follow-up intervals according to these risk factors may lead to better adherence of the families to the protocols and decrease the burden on those centers caring for these patients.

International Children's Continence Society classifies detrusor anomalies in the voiding phase as overactive and underactive (8). In the voiding phase, the inability of the urethral sphincter to relax due to neuropathic reasons has been defined as DSD. By the addition of cases with no sphincter activity, basically, four types of neuropathic bladder-sphincter disorders are revealed $(9,10)$. Various studies were conducted showing the possible negative effects of high detrusor pressure on kidney function. Steinhardt et al. (11) showed that in patients with SB, the glomerular filtration rate decreased when the bladder filling pressure was $35-40 \mathrm{cmH}_{2} \mathrm{O}$ (11). Austin et al. (12) highlighted that DLPP over $40 \mathrm{cmH}_{2} \mathrm{O}$ is a high value that can cause upper urinary system damage. The cases with overactive bladder and DSD would be the most vulnerable patients who have higher bladder pressure (13). For these reasons, DLPP over $40 \mathrm{cmH}_{2} \mathrm{O}, \mathrm{DSD}$, and high-pressure overactivity are defined as urodynamic "hostile bladder" parameters by the CDC (7).

There have been different studies to determine the threshold DLPP value that causes urinary tract damage. The threshold value that generally indicates the hostility of the bladder has been determined to be $40-50 \mathrm{cmH}_{2} \mathrm{O}(11,12)$. Intravesical pressure reaching high values during the filling phase will likely affect the upper urinary tract. Determining this threshold may help to guide treatment. Although this threshold value appeared to be $50 \mathrm{cmH}_{2} \mathrm{O}$ in our study, it might be safer to accept a lower threshold value as different results were found in different studies, and some of these were as low as $20 \mathrm{cmH}_{2} \mathrm{O}$ (14). Prospective large series are needed in order to find the answer to this question.

Tanaka et al. (4) found the renal scar rate in the first scintigraphic examination of children with SB to be $7.5 \%$. This rate seems to be lower than in our study. This may be due to unintentional sampling bias in our study as patients with more problems tend to comply better with longer follow-up periods.

Vesicoureteral reflux studies revealed existing renal scars to be a risk factor for developing new scars $(15,16)$. Our study showed similar results with having a scar in the first scan being an independent risk factor for new scar development. Hydronephrosis was observed in 8 (13.7\%) of our cases; six of these were high grade. Tanaka et al. (4) detected hydronephrosis in $44.1 \%$ of cases with SB, and $3.7 \%$ had high-grade hydronephrosis in the initial US examinations. This rate is quite high compared to the rate in our study. In our series, 6 of 8 cases with hydronephrosis in the first US developed new scars $(p<0.05)$. A high degree of HN was present in 5 of these six cases. High-grade $\mathrm{HN}$ was found to be a significant risk factor in terms of new scar development. Due to the sequelae dilatations observed in some patients, hydronephrosis in SB patients can sometimes be considered insignificant. Our study emphasizes the importance of high-grade hydronephrosis as a warning sign for further evaluation to prevent kidney damage.

Bladder augmentation is performed in cases with high bladder pressure, low capacity, incontinence, and upper tract damage despite appropriate management (17). The rate of patients undergoing bladder augmentation in pretreated patients varies between $5-17 \%$, while in untreated cases, this rate can rise to $41 \%(3,17-19)$. In our study, the bladder augmentation rate was $12.1 \%$, similar to these, and similar to that of American SB Centers (12.7\%) (19). In a study by Corona et al. (20), VUR and a DLPP of $40 \mathrm{cmH}_{2} \mathrm{O}$ or above at the end of the filling phase were defined as the factors that increase the need for bladder augmentation. In studies conducted by different researchers, VUR incidence in patients with SB was between $27-34 \%(6,21)$. The VUR incidence in our study was similar to other studies at 
24.1\%. The mean first DLPP of patients who underwent augmentation cystoplasty was higher than those patients who did not require this procedure; however, the difference was not significant. The presence of NDO in the first urodynamic examination emerged as an independent risk factor for the need for augmentation.

\section{Study Limitations}

The most important limitation of our study is its retrospective nature. Also, the lack of compliance with protocols caused many patients to be excluded from this study. This is mostly secondary to being a tertiary center with patients referred from other provinces and centers, to re-evaluate their management.

\section{Conclusion}

Loss of low-pressure reservoir function of the bladder in cases with SB can cause upper urinary tract damage. Therefore, "hostile bladder" was defined by CDC to predict patients at risk of kidney damage. Our study confirmed the detrimental effects of high pressure and detrusor-sphincter dyssynergia; however, the hostile bladder parameters were not sufficient to distinguish high-risk group patients. The presence of scars in the first scintigraphic evaluation, DLPP above $50 \mathrm{cmH}_{2} \mathrm{O}$, and the presence of hydronephrosis in the first ultrasound were found to be risk factors for renal deterioration. More frequent monitoring and detailed evaluation may be necessary for those patients with these risk factors. Prospective studies are required to design better management strategies individualized for each patient.

\section{Ethics}

Ethics Committee Approval: Institutional Ethical approval was obtained for the retrospective patient chart review (20-8T/26).

Informed Consent: Retrospective study.

Peer-review: Externally peer-reviewed.

\section{Authorship Contributions}

Concept: A.T., S.T., I.U., Design: A.T., S.T., I.U., Data Collection or Processing: A.T., E.A.T., Analysis or Interpretation: A.T., Supervision: S.T., I.U., Writing: A.T., S.T., E.A.T., I.U.

Conflict of Interest: No conflict of interest was declared by the authors.

Financial Disclosure: The authors declared that this study received no financial support.

\section{References}

1. Mandiracioğlu A, Ulman I, Lüleci E, Ulman C. The incidence and risk factors of neural tube defects in Izmir, Turkey: a nested case-control study. Turk J Pediatr 2004; 46:214-20.

2. Kessler TM, Lackner I, Kiss G, Rehder $\mathrm{P}$, Madersbacher $\mathrm{H}$. Early proactive management improves upper urinary tract function and reduces the need for surgery in patients with myelomeningocele. Neurourol Urodyn 2006; 25:758-62.

3. Kaefer M, Pabby A, Kelly M, Darbey M, Bauer SB. Improved bladder function after prophylactic treatment of the high risk neurogenic bladder in newborns with myelomeningocele. J Urol 1999; 162:1068-71.

4. Tanaka ST, Paramsothy P, Thibadeau J, et al. Baseline urinary tract Imaging in infants enrolled in the UMPIRE protocol for children with spina bifida. J Urol 2019; 201:1193-8.

5. Galloway NTM, Mekras JA, Helms M, Webster GD. An objective score to predict upper tract deterioration in myelodysplasia. J Urol 1991;145:535-7.

6. Ozel SK, Dokumcu Z, Akyildiz C, Avanoglu A, Ulman I. Factors Affecting Renal Scar Development in Children with Spina Bifida. Urol Int 2007; 79:133-6.

7. Routh JC, Cheng EY, Austin JC, et al. Design and methodological considerations of the centers for disease control and prevention urologic and renal protocol for the newborn and young child with spina bifida. J Urol 2016; 196:1728-34.

8. Tarcan T, Bauer S, Olmedo E, Khoshbin S, Kelly M, Darbey M. Long-term followup of newborns with myelodysplasia and normal urodynamic findings: Is followup necessary? J Urol 2001; 165:564-7.

9. Verpoorten C, Buyse GM. The neurogenic bladder: medical treatment. Pediatr Nephrol 2008; 23:717-25.

10. Docimo SG, Canning D, Khoury A, Salle JLP. The Kelalis--King-Belman Textbook of Clinical Pediatric Urology. 6th ed. CRC Press, Boca Raton, Florida, 2018.

11. Steinhardt GF, Goodgold HM, Samuels LD. The effect of intravesical pressure on glomerular filtration rate in patients with myelomeningocele. J Urol 1988; 140:1293-5.

12. Austin PF, Bauer SB, Bower $\mathrm{W}$, et al. The standardization of terminology of lower urinary tract function in children and adolescents: Update report from the standardization committee of the International Children's Continence Society. Neurourol Urodyn 2016; 35:471-81.

13. Madersbacher $\mathrm{H}$. The various types of neurogenic bladder dysfunction: an update of current therapeutic Concepts. Paraplegia 1990; 28:217-29.

14. Tarcan T, Sekerci CA, Akbal C, et al. Is $40 \mathrm{~cm} \mathrm{H} 2 \mathrm{O}$ detrusor leak point pressure cut-off reliable for upper urinary tract protection in children with myelodysplasia? Neurourol Urodyn 2017; 36:759-63

15. Soylu A, Demir BK, Türkmen M, et al. Predictors of renal scar in children with urinary infection and vesicoureteral reflux. Pediatr Nephrol 2008; 23:2227-32.

16. Naseer SR, Steinhardt GF. New renal scars in children with urinary tract infections, vesicoureteral reflux and voiding dysfunction: a prospective evaluation. J Urol 1997; 158:566-8. 
17. Snow-Lisy DC, Yerkes EB, Cheng EY. Update on urological management of spina bifida from prenatal diagnosis to adulthood. J Urol 2015; 194:288-96.

18. Edelstein RA, Bauer SB, Kelly MD, et al. The long-term urological response of neonates with myelodysplasia treated proactively with intermittent catheterization and anticholinergic therapy. J Urol 1995; 154:1500-4.

19. Routh JC, Joseph DB, Liu T, et al. Bladder reconstruction rates differ among centers participating in national spina bifida patient registry. I Urol 2018; 199:268-73.
20. Corona LE, Lee T, Marchetti K, et al. Urodynamic and imaging findings in infants with myelomeningocele may predict need for future augmentation cystoplasty. J Pediatr Urol 2019; 15:644. e1-5. doi: 10.1016/j.jpurol.2019.09.015.

21. Flood HD, Ritchey ML, Bloom DA, Huang C, McGuire E). Outcome of reflux in children with myelodysplasia managed by bladder pressure monitoring. I Urol 1994; 152:1574-7. 